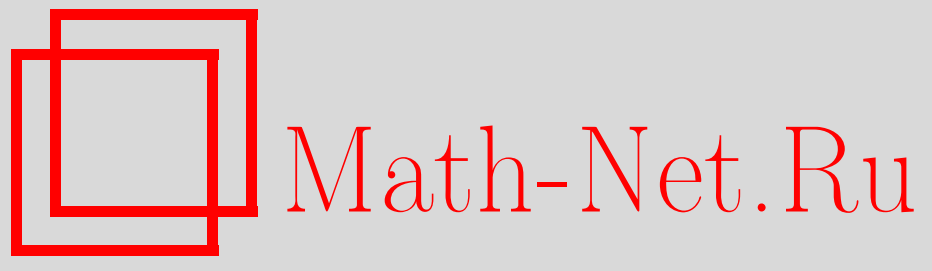

А. В. Калинкин, О вероятности вырождения ветвящегося процесса с двумя комплексами взаимодействия частиц, Теория вероятн. и ее примен., 2001, том 46, выпуск 2, 376-380

DOI: https://doi.org/10.4213/tvp3926

Использование Общероссийского математического портала MathNet.Ru подразумевает, что вы прочитали и согласны с пользовательским соглашением

http://www . mathnet.ru/rus/agreement

Параметры загрузки:

IP : 54.80 .73 .141

26 апреля 2023 г., 13:23:05

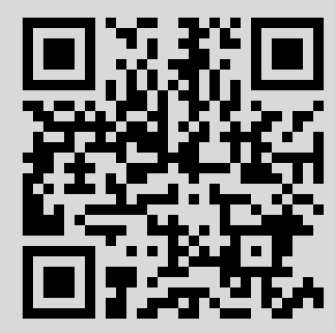


(c) $2001 \mathrm{r}$.

КАЛИНКИН А. В.*

\title{
О ВЕРОЯТНОСТИ ВЫРОЖДЕНИЯ ВЕТВЯЩЕГОСЯ ПРОЩЕССА С ДВУМЯ КОМПЛЕКСАМИ ВЗАИМОДЕЙСТВИЯ ЧАСТИЦ
}

\begin{abstract}
Рассматривается марковский ветвящийся процесс с непрерывным временем и одним типом частиц $T$, в котором любая пара частиц $T+T$, независимо от всех других частиц, порождает потомство. Кроме того, любая из частиц типа $T$ также порождает потомство. Получены выражения для вероятностей вырождения процесса при некоторых предположениях о распределении случайного числа потомков. Применяется метод экспоненциальной производящей функции для решения стационарной первой системы дифференциальных уравнений Колмогорова [6], [7].

Ключевые слова и фразы: ветвящийся процесс с взаимодействием частиц, вероятности вырождения, уравнение Лапласа для экспоненциальной производящей функции, точные решения.
\end{abstract}

1. Ветвящийся процесс с одним типом частиц $T$ и двумя комплексами взаимодействия $\varepsilon^{1}=1, \varepsilon^{2}=2$ [5]. На множестве состояний $\mathbf{N}=\{0,1,2, \ldots\}$ рассматривается однородный во времени марковский процесс $\xi_{t}, t \in[0, \infty)$, с переходными вероятностями $P_{i j}(t)=\mathbf{P}\left\{\xi_{t}=j \mid \xi_{0}=i\right\}, i, j \in \mathbf{N}$. Пусть при $\Delta t \rightarrow 0$ переходные вероятности имеют вид $(\lambda \geqslant 0, \mu \geqslant 0)$

$$
P_{i j}(\Delta t)= \begin{cases}\left(p_{j-i+2}^{2} i(i-1) \lambda+p_{j-i+1}^{1} i \mu\right) \Delta t+o(\Delta t), & \text { если } j \geqslant i-2, j \neq i ; \\ 1-(i(i-1) \lambda+i \mu) \Delta t+o(\Delta t), & \text { если } j=i ; \\ o(\Delta t), & \text { если } j \leqslant i-2,\end{cases}
$$

где $\left\{p_{l}^{k} \geqslant 0, l \in \mathbf{N} ; \sum_{l=0}^{\infty} p_{l}^{k}=1, p_{k}^{k}=0\right\}, k=1,2$, - заданные распределения вероятностей. Введем производящие функции

$$
F_{i}(t ; s)=\sum_{j=0}^{\infty} P_{i j}(t) s^{j}, \quad i \in \mathbf{N} ; \quad h_{k}(s)=\sum_{l=0}^{\infty} p_{l}^{k} s^{l}, \quad k=1,2 ;|s| \leqslant 1 .
$$

Вторая (прямая) система дифференциальных уравнений Колмогорова для процесса $\xi_{t}$ равносильна линейному уравнению в частных производных (см. [5, теоремa 1])

$$
\frac{\partial F_{i}(t ; s)}{\partial t}=\lambda\left(h_{2}(s)-s^{2}\right) \frac{\partial^{2} F_{i}(t ; s)}{\partial s^{2}}+\mu\left(h_{1}(s)-s\right) \frac{\partial F_{i}(t ; s)}{\partial s}
$$

с начальным условием $F_{i}(0 ; s)=s^{i}$.

Состояние $i$ интерпретируется как наличие $i$ частиц типа $T$. Можно полагать, что через случайные промежутки времени происходят взаимодействия частицы типа $T$ с другой частицей типа $T$. Эта пара частиц независимо от других частиц превращается в новую группу из $n$ частиц типа $T: T+T \rightarrow n T$, с распределением вероятностей $\left\{p_{n}^{2}\right\}$; процесс переходит в состояние $i+n-2$. Кроме того, через случайные промежутки времени, одна из частиц типа $T$ независимо от других частиц превращается в группу из $m$ частиц типа $T: T \rightarrow m T$, с распределением вероятностей $\left\{p_{m}^{1}\right\}$; процесс переходит в состояние $i+m-1$.

Процесс $\xi_{t}$ принадлежит специальному классу марковских процессов со счетным числом состояний, определенному Б. А. Севастьяновым [5]. В [1], [2] рассматриваемый процесс интерпретируется как модель химической реакции. Ж. Летесье и Г. Валэн построили точные незамкнутые решения уравнения (1) для различных частных случаев $h_{1}(s), h_{2}(s)$ в связи с приложениями в физике высоких энергий (см. [3] и др.).

* Московский государственный технический университет им. Н.Э. Баумана, кафедра высшей математики, 2-я Бауманская ул., 5, 107005 Москва, Россия; http://www.bmstu.ru/facult/fn/kalin/kalinkin.htm 
2. Задача о вероятностях вырождения. Для рассматриваемого марковского процесса $\xi_{t}$ состояние 0 является поглощающим. Вероятности вырождения вероятности попадания в поглощаюшее состояние - равны

$$
q_{i 0}=\lim _{t \rightarrow \infty} P_{i 0}(t), \quad i \in \mathbf{N} .
$$

Для обычного ветвящегося процесса с независимыми частицами, когда $\lambda=0, \mu>$ $0, q_{i 0}=q_{1}^{i}, i \in \mathbf{N}$, где $q_{1}-$ ближайший к нулю неотрицательный корень уравнения $h_{1}(s)-s=0$ (см. [4, гл. $\left.\left.2, \S 1\right]\right)$.

Для ветвящегося процесса с попарно взаимодействующими частицами, когда $\lambda>0, \mu=0\left(p_{0}^{2}>0\right)$,

$$
q_{i 0} \sim C_{0} q_{2}^{i}, \quad i \rightarrow \infty
$$

где $C_{0}>0$ и $q_{2}-$ ближайший к нулю неотрицательный корень уравнения $h_{2}(s)-s^{2}=0$ (см. [5], [7, следствие 1]).

Для ветвящегося процесса $\xi_{t}(\lambda>0, \mu>0)$ вводятся параметры критичности $m_{1}=\mu\left(h_{1}^{\prime}(1)-1\right), m_{2}=\lambda\left(h_{2}^{\prime}(1)-2\right)$ [4], [5]. Все возможные варианты значений этих параметров можно разделить на три случая: 1) $m_{2}>0$;2) $m_{2}=0, m_{1}>0$;) $m_{2}=0$, $m_{1} \leqslant 0$ или $m_{2}<0$.

В настоящей работе рассмотрен случай 1), получены выражения для вероятностей вырождения (2) при дополнительных предположениях о распределении числа потомков $\left\{p_{m}^{1}, m \in \mathbf{N}\right\},\left\{p_{n}^{2}, n \in \mathbf{N}\right\}$. Показано, что асимптотическое поведение вероятностей вырождения $q_{i 0}$ при $i \rightarrow \infty$ может быть отлично от вида (3).

В случае 3) можно предполагать, что $q_{i 0}=1$ для всех $i \in \mathbf{N}$.

3. Дифференциальное уравнение для экспоненциальной производящей функции вероятностей вырождения. Введем экспоненциальные производящие функции

$$
G_{j}(t ; z)=\sum_{i=0}^{\infty} \frac{z^{i}}{i !} P_{i j}(t), \quad j \in \mathbf{N} ; \quad g_{0}(z)=\sum_{i=0}^{\infty} \frac{z^{i}}{i !} q_{i 0},
$$

и линейные дифференциальные операторы

$$
h_{k}\left(\frac{d}{d z}\right)=\sum_{l=0}^{\infty} p_{l}^{k} \frac{d^{l}}{d z^{l}}, \quad k=1,2 .
$$

Первая (обратная) система дифференциальных уравнений Колмогорова для процесса $\xi_{t}$ равносильна линейному уравнению в частных производных ([5, теорема 2$]$; cp. [7, теорема 1])

$$
\frac{\partial G_{j}(t ; z)}{\partial t}=\left[\lambda z^{2}\left(h_{2}\left(\frac{\partial}{\partial z}\right)-\frac{\partial^{2}}{\partial z^{2}}\right)+\mu z\left(h_{1}\left(\frac{\partial}{\partial z}\right)-\frac{\partial}{\partial z}\right)\right] G_{j}(t ; z)
$$

с начальным условием $G_{j}(0 ; z)=z^{j} / j$ !.

Аналогично теореме 2 из [7] показывается, что $g_{0}(z)=\lim _{t \rightarrow \infty} G_{0}(t ; z)$ и $g_{0}(z)$ удовлетворяет стационарному первому уравнению Колмогорова

$$
\left[\lambda z\left(h_{2}\left(\frac{d}{d z}\right)-\frac{d^{2}}{d z^{2}}\right)+\mu\left(h_{1}\left(\frac{d}{d z}\right)-\frac{d}{d z}\right)\right] g_{0}(z)=0
$$

при граничном условии $g_{0}(0)=1$. Заметим, что $g_{0}(z)$ - целая функция порядка не больше единищы, так как

$$
\left|g_{0}(z)\right| \leqslant \sum_{i=0}^{\infty} \frac{|z|^{i}}{i !}\left|q_{i 0}\right| \leqslant e^{|z|}
$$


4. Об интегральном представлении для вероятностей вырождения $q_{\text {io }}$. Пусть $h_{1}(s), h_{2}(s)$ - многочлены. Обыкновенное дифференциальное уравнение (5) относится к типу уравнений Лапласа (см. [8, часть I, гл. 5, § 22.4]) и может быть решено методом определенного интеграла,

$$
g_{0}(z)=\int_{K} e^{z u} \varphi(u) d u
$$

где $\varphi(u)=\left(h_{2}(u)-u^{2}\right)^{-1} \exp \left((\mu / \lambda) \int_{u_{0}}^{u}\left[\left(h_{1}(v)-v\right) /\left(h_{2}(v)-v^{2}\right)\right] d v\right)$; причем кривая $K$ в комплексной $u$-плоскости удовлетворяет условию

$$
\int_{K} \frac{d}{d u} e^{z u}\left(h_{2}(u)-u^{2}\right) \varphi(u) d u=0 .
$$

Из определения (4) экспоненциальной производящей функции $g_{0}(z)$ и разложения $e^{z u}=\sum_{i=0}^{\infty} z^{i} u^{i} / i$ ! следует представление для вероятностей вырождения

$$
q_{i 0}=\int_{K} \frac{u^{i}}{h_{2}(u)-u^{2}} \exp \left(\frac{\mu}{\lambda} \int_{u_{0}}^{u} \frac{h_{1}(v)-v}{h_{2}(v)-v^{2}} d v\right) d u, \quad i \in \mathbf{N}
$$

Нахождение кривой $K$ представляет собой сложную задачу, связанную с рассмотрением римановой поверхности, соответствуюшей функции $\varphi(u)$ [8]. В настоящей работе получены $q_{i 0}$ для частных случаев $h_{1}(s), h_{2}(s)$.

Заметим, что функция $e^{z}$ является решением уравнения (5) при любых $h_{1}(s), h_{2}(s)$.

5. Интегральное представление для вероятностей вырождения $q_{\text {i0 }}$ в случае $h_{1}(s)=p_{2}^{1} s^{2}+p_{0}^{1}, h_{2}(s)=p_{3}^{2} s^{3}+p_{1}^{2} s+p_{0}^{2}, m_{2}>0$.

Лемма. Пусть $h_{1}(s)=p_{2}^{1} s^{2}+p_{0}^{1}, h_{2}(s)=p_{3}^{2} s^{3}+p_{1}^{2} s+p_{0}^{2}$, причем $p_{0}^{1}+p_{0}^{2}>0$, $m_{2}>0$ u $p_{0}^{2}+p_{1}^{2}>0$. Tогдa $\lim _{i \rightarrow \infty} q_{i 0}=0$.

Д ока 3 а т л л с т в о. Ограничимся рассмотрением случая $h_{2}(s)=p_{3}^{2} s^{3}+$ $p_{1}^{2} s$. Процесс $\xi_{t}$ может иметь скачки $-1,+1$. Если рассматривать процесс только в моменты изменения состояния, то получаем «вложенную цепь Маркова» $X_{0}, X_{1}, \ldots, X_{n}, \ldots$, для которой $(i \neq 0)$

$$
\begin{aligned}
& \mathbf{P}\left\{X_{n+1}=i-1 \mid X_{n}=i\right\}=\frac{p_{0}^{1} i \mu}{i \mu+i(i-1) \lambda}+\frac{p_{1}^{2} i(i-1) \lambda}{i \mu+i(i-1) \lambda} \\
& \mathbf{P}\left\{X_{n+1}=i+1 \mid X_{n}=i\right\}=\frac{p_{2}^{1} i \mu}{i \mu+i(i-1) \lambda}+\frac{p_{3}^{2} i(i-1) \lambda}{i \mu+i(i-1) \lambda} .
\end{aligned}
$$

Вероятность попадания цепи $X_{n}$ в поглощающее состояние 0 , при начальном состоянии $i$, совпадает с вероятностью вырождения $q_{i 0}$ для процесса $\xi_{t}$; имеем соотношение для вероятностей вырождения:

$$
q_{i 0}=\frac{p_{0}^{1} \mu+p_{1}^{2}(i-1) \lambda}{\mu+(i-1) \lambda} q_{i-1,0}+\frac{p_{2}^{1} \mu+p_{3}^{2}(i-1) \lambda}{\mu+(i-1) \lambda} q_{i+1,0} .
$$

Получаем линейное однородное разностное уравнение с линейными коэффициентами (см. [9, гл. $7, \S 46$, п. 5], [10, гл. 2, §5])

$$
\left(p_{3}^{2} \lambda(i+2)-2 p_{3}^{2} \lambda+p_{2}^{1} \mu\right) q_{i+2,0}-(\lambda(i+1)-\lambda+\mu) q_{i+1,0}+\left(p_{1}^{2} \lambda i+p_{0}^{1} \mu\right) q_{i 0}=0
$$

$i \in \mathbf{N}$, при начальном условии $q_{00}=1$.

Для решения последнего уравнения в [9] получена оценка

$$
\left|q_{i 0}\right| \leqslant C_{\varepsilon}\left(\frac{1}{R}-\varepsilon\right)^{i}, \quad i \in \mathbf{N},
$$

где $\varepsilon>0, C_{\varepsilon}>0$ - некоторая постоянная, $R=\min \left(\left|z_{1}\right|,\left|z_{2}\right|\right)$, где $z_{1}, z_{2}$ - корни уравнения $p_{1}^{2} z^{2}-z+p_{3}^{2}=0$. В условиях леммы $z_{1}=1, z_{2}=p_{3}^{2} / p_{1}^{2}>1$ и $R=1$. Из (8) следует утверждение леммы.

Аналогично, с использованием методов [9], рассматривается случай $h_{2}(s)=$ $p_{3}^{2} s^{3}+p_{1}^{2} s+p_{0}^{2}$. Лемма доказана. 
Уравнение (5) для экспоненциальной производящей функции вероятностей вырождения $g_{0}(z)$ в рассматриваемом случае получает вид

$$
\lambda p_{3}^{2} z g_{0}^{\prime \prime \prime}(z)+\left(-\lambda z+\mu p_{2}^{1}\right) g_{0}^{\prime \prime}(z)+\left(\lambda p_{1}^{2} z-\mu\right) g_{0}^{\prime}(z)+\left(\lambda p_{0}^{2} z+\mu p_{0}^{1}\right) g_{0}(z)=0 .
$$

При сделанных предположениях имеем

$$
h_{1}(s)-s=p_{2}^{1}(s-1)\left(s-q_{1}\right), \quad h_{2}(s)-s^{2}=p_{3}^{2}(s-1)\left(s-q_{2}\right)\left(s-\widehat{q}_{2}\right) .
$$

Условия $m_{1}>0$ и $p_{0}^{1}>0$ равносильны условию $0<q_{1}<1$. Также нетрудно проверить, что из условий $m_{2}>0$ и $p_{0}^{2}+p_{1}^{2}>0$ следует $0<q_{2}<1$ и $-q_{2}<\widehat{q}_{2} \leqslant 0$ [7].

Теорема 1. Пусть $h_{1}(s)=p_{2}^{1} s^{2}+p_{0}^{1}, h_{2}(s)=p_{3}^{2} s^{3}+p_{1}^{2} s+p_{0}^{2}$, причем $m_{2}>0$ и $p_{0}^{2}+p_{1}^{2}>0$. Пусть $q_{1}=q_{2}$. Тогда вероятности вырождения ветвящегося

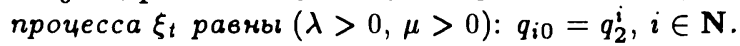

Д ока $з$ а т л в с т в о. Находим решение уравнения (9) при условии (6) и $g_{0}(0)=1$. Для уравнения (9) точка $z=0$ является слабо особой точкой (см. [8, гл. 5 , $\S 18.2, \S 18.6(\mathrm{a})])$. Следуя [8], можно показать, что для линейного дифференциального уравнения третьего порядка (9) не существует трех линейно независимых решений таких, чтобы каждое из них было аналитическим на всей комплексной $z$-плоскости. Непосредственной подстановкой в уравнение (9) функций $e^{q_{2} z}$ и $e^{z}$ убеждаемся, что они являются частными решениями. Следовательно, в силу условия (6), искомое решение $g_{0}(z)$ имеет вид $g_{0}(z)=C_{1} e^{q_{2} z}+C_{2} e^{z}$, где $C_{1}, C_{2}$ - некоторые константы. Отсюда получаем $q_{i 0}=C_{1} q_{2}^{i}+C_{2}$, и $\lim _{i \rightarrow \infty} q_{i 0}=C_{2}$. По лемме $C_{2}=0$. Из условия $\jmath_{0}(0)=1$ получаем окончательно $g_{0}(z)=e^{q_{2} z}$. Теорема 1 доказана.

Теорема 2. Пусть $h_{1}(s)=p_{2}^{1} s^{2}+p_{0}^{1}, h_{2}(s)=p_{3}^{2} s^{3}+p_{1}^{2} s+p_{0}^{2}$, причем $p_{0}^{1}+p_{0}^{2}>0$, $m_{2}>0 u p_{0}^{2}+p_{1}^{2}>0$. Положим

$$
\alpha=\frac{\mu}{\lambda} \cdot \frac{h_{1}\left(q_{2}\right)-q_{2}}{h_{2}^{\prime}\left(q_{2}\right)-2 q_{2}}, \quad \beta=\frac{\mu}{\lambda} \cdot \frac{h_{1}\left(\widehat{q}_{2}\right)-\widehat{q}_{2}}{h_{2}^{\prime}\left(\widehat{q}_{2}\right)-2 \widehat{q}_{2}},
$$

и пусть $\alpha>0$. Вероятности вырождения ветвяшегося прочесса $\xi_{t}$ равнь $(\lambda>0$, $\mu>0$ )

$$
q_{\mathrm{i} 0}=\frac{1}{A} \int_{\widehat{q}_{2}}^{q_{2}} \frac{u^{i}\left(q_{2}-u\right)^{\alpha-1}\left(u-\widehat{q}_{2}\right)^{\beta-1}}{p_{3}^{2}(1-u)} d u, \quad i \in \mathbf{N},
$$

где константа $A$ определяется условием $q_{00}=1$

$$
A=\int_{\widehat{q}_{2}}^{q_{2}} \frac{\left(q_{2}-u\right)^{\alpha-1}\left(u-\widehat{q}_{2}\right)^{\beta-1}}{p_{3}^{2}(1-u)} d u .
$$

Д оказа те льс тво. Найдем решение уравнения (9) при условии (6) и $g_{0}(0)=1$. Следуя [8] (часть III, уравнение (5.7)), вычисляем $\left(\widehat{q}_{2}<u_{0}<q_{2}\right)$

$$
\begin{aligned}
\varphi(u) & =\frac{1}{p_{3}^{2}(u-1)\left(u-q_{2}\right)\left(u-\widehat{q}_{2}\right)} \exp \left(\frac{\mu}{\lambda} \int_{u_{0}}^{u} \frac{p_{2}^{1}(v-1)\left(v-q_{1}\right)}{p_{3}^{2}(v-1)\left(v-q_{2}\right)\left(v-\widehat{q}_{2}\right)} d v\right) \\
& =C_{0} \cdot \frac{\left(q_{2}-u\right)^{\alpha-1}\left(u-\widehat{q}_{2}\right)^{\beta-1}}{p_{3}^{2}(u-1)}, \quad C_{0}=\frac{1}{\left(q_{2}-u_{0}\right)^{\alpha}\left(u_{0}-\widehat{q}_{2}\right)^{\beta}},
\end{aligned}
$$

где $\alpha$ и $\beta$ определены формулами (11). Подставляя в (11) выражения (10), получаем

$$
\alpha=\frac{\mu}{\lambda} \cdot \frac{p_{2}^{1}\left(q_{2}-q_{1}\right)}{p_{3}^{2}\left(q_{2}-\widehat{q}_{2}\right)}, \quad \beta=\frac{\mu}{\lambda} \cdot \frac{p_{2}^{1}\left(q_{1}-\widehat{q}_{2}\right)}{p_{3}^{2}\left(q_{2}-\widehat{q}_{2}\right)} .
$$

Таким образом, $\beta>0$.

В качестве кривой интегрирования $K$ в формуле (7) можно взять, при условиях $\alpha>0, \beta>0$, отрезок между корнями $\widehat{q}_{2}$ и $q_{2}$ уравнения $h_{2}(s)-s^{2}=0$ (см. [8, часть I, гл. $5, \S 19.2])$, последний абзац пункта (Б); получаем частное решение уравнения (9),

$$
\int_{\widehat{q}_{2}}^{q_{2}} e^{z u} \frac{\left(q_{2}-u\right)^{\alpha-1}\left(u-\widehat{q}_{2}\right)^{\beta-1}}{p_{3}^{2}(1-u)} d u
$$

(что проверяется подстановкой последнего выражения в уравнение (9)). 
Это частное решение является аналитическим в комплексной $z$-плоскости (ср. далее вывод следствия). Дифференциальное уравнение третьего порядка (9) не имеет трех линейно независимых аналитических решений; в силу условия (6)

$$
g_{0}(z)=C_{1} \int_{\widehat{q}_{2}}^{q_{2}} e^{z u} \frac{\left(q_{2}-u\right)^{\alpha-1}\left(u-\widehat{q}_{2}\right)^{\beta-1}}{p_{3}^{2}(1-u)} d u+C_{2} e^{z}
$$

где $C_{1}, C_{2}$ - некоторые константы. Так же, как при доказательстве теоремы 1 , показывается, что $C_{2}=0$. Учитывая граничное условие $g_{0}(0)=1$, получаем формулу (12). Теорема 2 доказана.

Вычислим значение константы $A$. После замены переменной $x=\left(u-\widehat{q}_{2}\right) /\left(q_{2}-\widehat{q}_{2}\right)$ получаем

$$
\begin{aligned}
A & =\frac{\left(q_{2}-\widehat{q}_{2}\right)^{\alpha+\beta-1}}{p_{3}^{2}\left(1-\widehat{q}_{2}\right)} \int_{0}^{1} \frac{x^{\beta-1}(1-x)^{\alpha-1}}{1-x c} d x \\
& =\frac{\left(q_{2}-\widehat{q}_{2}\right)^{\alpha+\beta-1}}{p_{3}^{2}\left(1-\widehat{q}_{2}\right)} \frac{\Gamma(\alpha) \Gamma(\beta)}{\Gamma(\alpha+\beta)} F(1, \beta ; \alpha+\beta ; c),
\end{aligned}
$$

где

$$
c=\frac{q_{2}-\widehat{q}_{2}}{1-\widehat{q}_{2}}<1
$$

$\Gamma(\alpha)$ - гамма-функция, $F(\alpha, \beta ; \gamma ; c)$ - гипергеометрическая функция.

Та же замена переменной для интеграла (12) дает

$$
q_{i 0}=\frac{\Gamma(\alpha+\beta)}{\Gamma(\alpha) \Gamma(\beta) F(1, \beta ; \alpha+\beta ; c)} \int_{0}^{1} \frac{\left(\left(q_{2}-\widehat{q}_{2}\right) x+\widehat{q}_{2}\right)^{i} x^{\beta-1}(1-x)^{\alpha-1}}{1-c x} d x
$$

Применив к последнему интегралу стандартные методы получения асимптотических формул при $i \rightarrow \infty$ (см., например, [11, теорема 16.1]), получаем

Следствие. При $i \rightarrow \infty$ выполявется сооткоиекие

$$
q_{i 0} \sim C \cdot \frac{q_{2}^{i}}{i^{\alpha}}, \quad C=\frac{1}{1-c} \cdot \frac{q_{2}^{\alpha}}{\left(q_{2}-\widehat{q}_{2}\right)^{\alpha}} \cdot \frac{\Gamma(\alpha+\beta)}{\Gamma(\beta) F(1, \beta ; \alpha+\beta ; c)} .
$$

Отметим, что при $\alpha=0$ (тогда, в силу равенств (13), $q_{1}=q_{2}$ ) $C=1$.

\section{СПИСОК ЛИТЕРАТУРЫ}

1. Баруча-Рид A. Т. Элементы теории марковских процессов и их приложения. М.: Наука, 1969,512 с.

2. McQuarrie D. A., Jachimowcki C.J., Russel M.E. Kinetic of small system. II. J. Chem. Phys., 1964, v. 40, № 10, p. 2914-2921.

3. Letessier J., Valent $G$. The generating function method for quadratic asimptotically symmetric birth and death processes. - SIAM J. Appl. Math., 1984, v. 44, p. 773783.

4. Севастьяяов Б. А. Ветвящиеся пронессы. М.: Наука, 1971, 436 с.

5. Севастьяяов Б. А., Калиякия $A . B$. Ветвящиеся случайные процессы с взаимодействием частиц. - Докл. АН СССР, 1982, т. 264, в. 2, с. 306-308.

6. Калиякия $A . B$. Вероятность вырождения одного ветвящегося процесса. - Некоторые вопросы математики и механики. Под ред. В.В. Козлова и Б. В. Шабата. М.: Изд-во МГУ, 1983, с. 58-59.

7. Калиякин $A$. B. Вероятность вырождения ветвящегося процесса с взаимодействием частиц. - Теория вероятн. и ее примен., 1982, т. 27, в. 1, с. 192-197.

8. Камке Э. Справочник по обыкновенным дифференциальным уравнениям. М.: Наука, $1971,576 \mathrm{c}$

9. Сидоров Ю. В., Федорюх М. В., Шабуния М. И. Лекции по теории функций комплексного переменного. М.: Наука, 1989,480 с.

10. Миролюбов $A . A$., Солдатов $M . A$. Линейные однородные разностные уравнения. M.: Наука, 1981, 208 с. 